Interfaces and Free Boundaries 12 (2010), 527-549

DOI $10.4171 / \mathrm{IFB} / 244$

\title{
Mass conserving Allen-Cahn equation and volume preserving mean curvature flow
}

\author{
XINFU CHEN \\ Department of Mathematics, University of Pittsburgh, Pittsburgh, PA 15260, USA \\ E-mail:xinfu@pitt.edu \\ D. HILHORST \\ Laboratoire de Mathématiques, Analyse Numérique \\ and EDP, CNRS (UMR 8628) et Université de Paris-Sud, 91405 Orsay Cedex, France \\ E-mail: danielle.hilhorst@math.u-psud.fr \\ E. LOGAK \\ CNRS (UMR 8088) and Department of Mathematics, Université de Cergy-Pontoise, \\ 2 rue A. Chauvin, 95302 Cergy-Pontoise Cedex, France \\ E-mail: Elisabeth.Logak@u-cergy.fr
}

[Received 22 April 2009 and in revised form 11 June 2010]

We dedicate this article to the memory of Michelle Schatzman

\begin{abstract}
We consider a mass conserving Allen-Cahn equation $u_{t}=\Delta u+\varepsilon^{-2}(f(u)-\varepsilon \lambda(t))$ in a bounded domain with no flux boundary condition, where $\varepsilon \lambda(t)$ is the average of $f(u(\cdot, t))$ and $-f$ is the derivative of a double equal well potential. Given a smooth hypersurface $\gamma_{0}$ contained in the domain, we show that the solution $u^{\varepsilon}$ with appropriate initial data tends, as $\varepsilon \searrow 0$, to a limit which takes only two values, with the jump occurring at the hypersurface obtained from the volume preserving mean curvature flow starting from $\gamma_{0}$.
\end{abstract}

\section{Introduction}

In this paper, we study the limit, as $\varepsilon \rightarrow 0$, of the solution $u^{\varepsilon}$ to the mass conserving Allen-Cahn equation $\left(P^{\varepsilon}\right)$

$$
\left(P^{\varepsilon}\right) \begin{cases}u_{t}^{\varepsilon}=\Delta u^{\varepsilon}+\varepsilon^{-2}\left(f\left(u^{\varepsilon}\right)-f_{\Omega} f\left(u^{\varepsilon}\right)\right) & \text { in } \Omega \times \mathbb{R}^{+}, \\ \partial_{\nu} u^{\varepsilon}=0 & \text { on } \partial \Omega \times \mathbb{R}^{+}, \\ u^{\varepsilon}(\cdot, 0)=g^{\varepsilon}(\cdot) & \text { on } \Omega \times\{0\},\end{cases}
$$

where

$$
f_{\Omega} f\left(u^{\varepsilon}\right)=\frac{1}{|\Omega|} \int_{\Omega} f\left(u^{\varepsilon}(x, t)\right) \mathrm{d} x .
$$

Here $\Omega$ is a smooth bounded domain in $\mathbb{R}^{n}(n \geqslant 1), \partial_{v}$ the outward normal derivative to $\partial \Omega$, and $-f(u)$ is the derivative of a smooth double-well potential with wells of equal depths; more precisely,

$$
f \in C^{\infty}(\mathbb{R}), \quad f( \pm 1)=0, \quad f^{\prime}( \pm 1)<0, \quad \int_{-1}^{u} f=\int_{1}^{u} f<0 \quad \forall u \in(-1,1) .
$$

A typical example is $f(u)=u-u^{3}$. 
Problem (1) was proposed, along with its well-posedness, by Rubinstein and Sternberg [22] as a model for phase separation in binary mixture. Note that the well-posedness of (1) follows from the general theory of semilinear parabolic equations [18]. The model is mass conserving and energy decreasing since

$$
\forall t \geqslant 0, \quad \frac{\mathrm{d}}{\mathrm{d} t} \int_{\Omega} u^{\varepsilon}(x, t) \mathrm{d} x=0
$$

and

$$
\forall t \geqslant 0, \quad \frac{\mathrm{d}}{\mathrm{d} t} \int_{\Omega}\left(\frac{\varepsilon\left|\nabla u^{\varepsilon}\right|^{2}}{2}+\frac{1}{\varepsilon} F\left(u^{\varepsilon}\right)\right) \mathrm{d} x=-\varepsilon \int_{\Omega}\left(u_{t}^{\varepsilon}\right)^{2} \mathrm{~d} x \leqslant 0,
$$

where $F(u):=-\int_{-1}^{u} f(s) \mathrm{d} s$ is the double equal well potential.

Formally, one can show that, as $\varepsilon \rightarrow 0$, assuming (6), the solution $u^{\varepsilon}$ to (1) tends to a limit given by

$$
\lim _{\varepsilon \rightarrow 0} u^{\varepsilon}(x, t)= \begin{cases}-1, & x \in \Omega_{t}, \\ +1, & x \in \Omega \backslash \bar{\Omega}_{t},\end{cases}
$$

where $\Omega_{t} \subset \subset \Omega, \gamma_{t}=\partial \Omega_{t}$ and $\Gamma:=\bigcup_{t \geqslant 0}\left(\gamma_{t} \times\{t\}\right)$ is the solution to the volume preserving mean curvature motion equation

$$
V=-(n-1) K_{\gamma_{t}}+\frac{n-1}{\left|\gamma_{t}\right|} \int_{\gamma_{t}} K_{\gamma_{t}} \mathrm{~d} H^{n-1} \quad \text { on } \gamma_{t} .
$$

Here $V$ is the normal velocity of $\gamma_{t}$ (negative when $\gamma_{t}$ is shrinking) and $K_{\gamma_{t}}$ the mean curvature (positive at points around which $\Omega_{t}$ is locally convex). Note that the integral of the curvature on $\gamma_{t}$ is the sum of the integrals of the curvature on each of the (finitely many) connected components of $\gamma_{t}$, with the orientation given by the outer normal vector on $\gamma_{t}$.

The local in time existence of a unique smooth solution to (4) has been first established in a two-dimensional setting in [12]. The general result in arbitrary space dimension is obtained in [14], where the large time behavior of solutions for initial data close to a sphere was also investigated. When the initial data is convex, it is shown in [19] that (4) admits a unique global in time convex solution. Related properties of other volume-preserving curvature driven flows are established in [13]. In particular it is shown that the solution to (4) will develop singularities in finite time. This was previously established in the case of multiple interfaces in the radial setting in [5].

Concerning the connection between (1) and (4), Bronsard and Stoth [5] considered a radially symmetric case with multiple interfaces (rings) and proved (3). The combination of energy and viscosity methods allowed the authors in [5] to study the convergence of the evolution problem $\left(P^{\varepsilon}\right)$ even after the formation of singularities, defining "ghost" or "phantom" interfaces. Let us also mention [17] where the Rubinstein-Sternberg model is modified in order to ensure that the solution $u^{\varepsilon}$ satisfies $\left|u^{\varepsilon}\right| \leqslant 1$. This allows the author to use the method introduced in [3] and to prove convergence to Problem (4).

In this paper we prove the convergence result stated in 3 under the following assumptions about the initial data.

There exists a smooth subdomain $\Omega_{0} \subset \subset \Omega$ such that $\gamma_{0}=\partial \Omega_{0}$ is a smooth hypersurface without boundary with finitely many connected components. 
Once $\gamma^{0}$ satisfies this assumption, we will construct $g^{\varepsilon}$ satisfying

$$
\lim _{\varepsilon \rightarrow 0} g^{\varepsilon}(x)= \begin{cases}-1, & x \in \Omega_{0}, \\ +1, & x \in \Omega \backslash \bar{\Omega}_{0} .\end{cases}
$$

We establish the following result.

THEOREm 1 Assume that $\gamma_{0}$ satisfies (5). Let $\Gamma=\bigcup_{0 \leqslant t \leqslant T}\left(\gamma_{t} \times\{t\}\right)$ be a smooth solution to (4) such that $\gamma_{t} \subset \subset \Omega$ for all $t \in[0, T]$. Then there exists a family of continuous functions $\left\{g^{\varepsilon}\right\}_{0<\varepsilon \leqslant 1}$ satisfying (6) such that the solution $u^{\varepsilon}$ to (1) satisfies (3) for all $t \in[0, T]$.

For the Allen-Cahn equation $u_{t}^{\varepsilon}=\Delta u^{\varepsilon}-\varepsilon^{-2} f\left(u^{\varepsilon}\right)$, 3 holds with $\Gamma$ being the solution to the motion by mean curvature flow $V=-(n-1) K_{\gamma_{t}}$. A simple method to verify this is to use a comparison principle and construct sub-super solutions [6, 16]. There are different notions of weak solutions such as viscosity [16] and varifold [20] solutions which can be used to establish the global in time limit. Nevertheless, (1) does not have a comparison principle (due to the volume preserving property) and the simple method does not seem to work. Here we shall employ a method first used by de Mottoni and Schatzman [11] for the Allen-Cahn equation, and later on by Alikakos, Bates, and Chen [1] for the Cahn-Hillard equation and Caginalp and Chen [8] for the phase field system. Namely we first rewrite the equation for $u^{\varepsilon}$ in Problem $\left(P^{\varepsilon}\right)$ as

$$
u_{t}^{\varepsilon}=\Delta u^{\varepsilon}+\varepsilon^{-2}\left(f\left(u^{\varepsilon}\right)-\varepsilon \lambda_{\epsilon}(t)\right) \quad \text { in } \Omega \times \mathbb{R}^{+},
$$

where we define

$$
\forall t \geqslant 0, \quad \lambda_{\epsilon}(t)=\frac{1}{\varepsilon} f_{\Omega} f\left(u^{\varepsilon}(\cdot, t)\right) .
$$

The basic strategy of the proof is as follows [1].

1. For a large enough $k \in \mathbb{N}$, construct an approximate solution $\left(u_{k}^{\varepsilon}, \lambda_{k}^{\varepsilon}\right)$ satisfying

$$
\left\{\begin{array}{l}
\left(u_{k}^{\varepsilon}\right)_{t}-\Delta u_{k}^{\varepsilon}-\varepsilon^{-2}\left(f\left(u_{k}^{\varepsilon}\right)-\varepsilon \lambda_{k}^{\varepsilon}\right)=\delta_{k}^{\varepsilon} \quad \text { in } \Omega_{T}:=\Omega \times[0, T], \\
\int_{\Omega}\left(u_{k}^{\varepsilon}\right)_{t} \mathrm{~d} x=0 \quad \forall t \in[0, T], \quad \partial_{\nu} u_{k}^{\varepsilon}=0 \quad \text { on } \partial \Omega \times[0, T],
\end{array}\right.
$$

where $\delta_{k}^{\varepsilon}=O(1) \varepsilon^{k}$. Note that, by integration,

$$
\varepsilon \lambda_{k}^{\varepsilon}(t)=f_{\Omega} f\left(u_{k}^{\varepsilon}\right)+O(1) \varepsilon^{k+2} .
$$

2. For each $t \in[0, T]$ and small $\varepsilon>0$, estimate the lower bound of the spectrum of the self-adjoint operator $-\Delta-\varepsilon^{-2} f^{\prime}\left(u_{k}^{\varepsilon}(\cdot, t)\right)$; namely, show that for some positive constant $C^{*}$,

$$
\inf _{0<t \leqslant T} \inf _{0<\varepsilon \leqslant 1} \inf _{\int_{\Omega} \phi=0, \int_{\Omega} \phi^{2}=1} \int_{\Omega}\left(|\nabla \phi|^{2}-\varepsilon^{-2} f^{\prime}\left(u_{k}^{\varepsilon}(\cdot, t)\right) \phi^{2}\right) \geqslant-C^{*} .
$$

3. Set $R=u^{\varepsilon}-u_{k}^{\varepsilon}$ and show that $R$ tends to 0 as $\varepsilon \rightarrow 0$.

Note that our analysis establishes the convergence as long as a smooth solution to the limit problem (4) exists, before the formation of singularities.

The organization of the paper is as follows. In Section 2, we present an error estimate required in step 3. In Section 3, we recall a known spectrum estimate [10, 7] that can be adapted here to prove step 2 in the strategy described above. After some preliminary geometrical computations in Section 4, we finally construct the approximate solution in Section 5. 


\section{Error estimate}

The error estimate relies on the following result which is proved in the appendix.

LEMMA 1 Let $\Omega \subset \mathbb{R}^{n}$ (with $n \geqslant 1$ ) be a bounded domain. Let $p=\min \{4 / n, 1\}$. Then there exists $C=C_{n}(\Omega)>0$ such that for every $R \in H^{1}(\Omega)$ with $\int_{\Omega} R \mathrm{~d} x=0$,

$$
\|R\|_{L^{2+p}}^{2+p} \leqslant C\|R\|_{L^{2}}^{p}\|\nabla R\|_{L^{2}}^{2}
$$

where $L^{q}=L^{q}(\Omega)$ for any $q \geqslant 1$.

Rubinstein-Sternberg [22] established $L^{\infty}$ bounds for the solution $u^{\varepsilon}$ to Problem $\left(P^{\varepsilon}\right)$ using invariant rectangles. Therefore we can modify $f$ outside a compact interval and assume for simplicity that

$$
\lim _{u \rightarrow \pm \infty} f(u)=\mp \infty
$$

and that there exists $M>0$ such that

$$
\forall|u| \geqslant M, \quad u f^{\prime \prime}(u) \leqslant 0 .
$$

Since $p \in(0,1]$, for any $C_{0}>0$ there exists $C=C\left(C_{0}, p\right)$ such that for all $|u| \leqslant C_{0}$ and $R \in \mathbb{R}$,

$$
\left(f(u+R)-f(u)-f^{\prime}(u) R\right) R \leqslant C|R|^{p+2} .
$$

Indeed, note that for $R$ in a compact interval, there is $\theta \in(0,1)$ such that

$$
\left(f(u+R)-f(u)-f^{\prime}(u) R\right) R=\frac{f^{\prime \prime}(u+\theta R)}{2} R^{3} \leqslant C|R|^{p+2},
$$

whereas for $|R| \rightarrow+\infty, f(u+R) R \rightarrow-\infty$ uniformly in $|u| \leqslant C_{0}$ so that

$$
\left(f(u+R)-f(u)-f^{\prime}(u) R\right) R \leqslant\left(-f(u)-f^{\prime}(u) R\right) R \leqslant C R^{2} \leqslant C|R|^{p+2} .
$$

LEMMA 2 Assume that $k>\max \{4, n\}$ and $\left\{u_{k}^{\varepsilon}\right\}_{0<\varepsilon \leqslant 1}$ satisfies $(9\}$ and $(10)$ with

$$
\left\|\delta_{k}^{\varepsilon}\right\|_{L^{2}\left(\Omega_{T}\right)} \leqslant \varepsilon^{k}, \quad\left\|u_{k}^{\varepsilon}\right\|_{L^{\infty}\left(\Omega_{T}\right)} \leqslant 2 .
$$

Let $\left\{u^{\varepsilon}\right\}_{0<\varepsilon \leqslant 1}$ be solutions to (1) with initial data $\left\{g^{\varepsilon}\right\}$ satisfying

$$
g^{\varepsilon}(\cdot)=u_{k}^{\varepsilon}(\cdot, 0)+\phi^{\varepsilon}(\cdot), \quad \int_{\Omega} \phi^{\varepsilon}=0, \quad\left\|\phi^{\varepsilon}\right\|_{L^{2}(\Omega)} \leqslant \varepsilon^{k} .
$$

Then for all sufficiently small positive $\varepsilon$,

$$
\sup _{0 \leqslant t \leqslant T}\left\|u^{\varepsilon}(\cdot, t)-u_{k}^{\varepsilon}(\cdot, t)\right\|_{L^{2}(\Omega)} \leqslant C(T) \varepsilon^{k} .
$$

REMARK 1 By a bootstrap argument using inequality (11), one can show that other norms of $u^{\varepsilon}-u_{k}^{\varepsilon}$ tend to 0 as $\varepsilon \searrow 0$. 
Proof. In the following, $C>0$ denotes a generic strictly positive constant independent of $\varepsilon>0$. Set $p=\min \{4 / n, 1\}$ and $R=u^{\varepsilon}-u_{k}^{\varepsilon}$. Then $\int_{\Omega} R(x, t) \mathrm{d} x=0$ for all $t \in[0, T]$. Also,

$$
R\left\{f\left(u^{\varepsilon}\right)-f\left(u_{k}^{\varepsilon}\right)-f^{\prime}\left(u_{k}^{\varepsilon}\right) R\right\} \leqslant C|R|^{2+p} .
$$

Multiplying by $R$ the difference of the equations for $u^{\varepsilon}$ and $u_{k}^{\varepsilon}$ and integrating the resulting equation over $\Omega$ gives, after integration by parts,

$$
\frac{1}{2} \frac{\mathrm{d}}{\mathrm{d} t}\|R\|_{L^{2}}^{2}+\int_{\Omega}\left\{|\nabla R|^{2}-\varepsilon^{-2} f^{\prime}\left(u_{k}^{\varepsilon}\right) R^{2}\right\} \leqslant \int_{\Omega}\left\{C \varepsilon^{-2}|R|^{2+p}+\left|R \delta_{k}^{\varepsilon}\right|\right\} .
$$

By $(10)$,

$$
\begin{aligned}
\int_{\Omega}\left\{|\nabla R|^{2}-\varepsilon^{-2}\right. & \left.f^{\prime}\left(u_{k}^{\varepsilon}\right) R^{2}\right\} \\
& =\varepsilon^{2} \int_{\Omega}\left\{|\nabla R|^{2}-\varepsilon^{-2} f^{\prime}\left(u_{k}^{\varepsilon}\right) R^{2}\right\}+\left(1-\varepsilon^{2}\right) \int_{\Omega}\left\{|\nabla R|^{2}-\varepsilon^{-2} f^{\prime}\left(u_{k}^{\varepsilon}\right) R^{2}\right\} \\
& \geqslant \varepsilon^{2}\|\nabla R\|_{L^{2}}^{2}-C\|R\|_{L^{2}}^{2} .
\end{aligned}
$$

The interpolation estimate (11) then yields

$$
\frac{1}{2} \frac{\mathrm{d}}{\mathrm{d} t}\|R\|_{L^{2}}^{2} \leqslant C\left\|\delta_{k}^{\varepsilon}\right\|_{L^{2}}\|R\|_{L^{2}}+C\|R\|_{L^{2}}^{2}-\|\nabla R\|_{L^{2}}^{2}\left\{\varepsilon^{2}-C_{1} \varepsilon^{-2}\|R\|_{L^{2}}^{p}\right\} .
$$

We define

$$
T_{\varepsilon}:=\sup \left\{t \in[0, T]:\|R(\cdot, \tau)\|_{L^{2}} \leqslant \varepsilon^{4 / p} C_{1}^{-1 / p} \text { for all } \tau \in[0, t]\right\} .
$$

Since $k>\max \{n, 4\}=4 / p$, it follows that

$$
\|R(\cdot, 0)\|_{L^{2}} \leqslant \varepsilon^{k}<\varepsilon^{4 / p} C_{1}^{-1 / p}
$$

for $\varepsilon>0$ small enough. Therefore, $T_{\varepsilon}>0$. Also, from [13), we have, for all $t \in\left(0, T_{\varepsilon}\right]$,

$$
\frac{\mathrm{d}}{\mathrm{d} t}\|R\|_{L^{2}} \leqslant C\left(\|R\|_{L^{2}}+\left\|\delta_{k}^{\varepsilon}\right\|_{L^{2}}\right) .
$$

Then Gronwall's inequality shows that

$$
\sup _{0 \leqslant t \leqslant T_{\varepsilon}}\|R(\cdot, t)\|_{L^{2}} \leqslant e^{C T_{\varepsilon}}\left[\|R(\cdot, 0)\|_{L^{2}}+C \int_{0}^{T_{\varepsilon}}\left\|\delta_{k}^{\varepsilon}\right\|_{L^{2}} \mathrm{~d} t\right] \leqslant C\left(T_{\varepsilon}\right) \varepsilon^{k} .
$$

Since for $\varepsilon>0$ small enough

$$
C\left(T_{\varepsilon}\right) \varepsilon^{k}<\frac{1}{2} \varepsilon^{4 / p} C_{1}^{-1 / p},
$$

we must have $T_{\varepsilon}=T$. This completes the proof. 


\section{The linearized operator}

\subsection{A spectrum estimate}

Assume that $f$ satisfies 22 . Then there is a unique solution $\theta_{0}: \mathbb{R} \rightarrow(0,1)$ to

$$
\theta_{0}^{\prime \prime}+f\left(\theta_{0}\right)=0 \quad \text { on } \mathbb{R}, \quad \theta_{0}( \pm \infty)= \pm 1, \quad \theta_{0}(0)=0 .
$$

The solution satisfies, for $\alpha=\min \left\{\sqrt{-f^{\prime}(1)}, \sqrt{-f^{\prime}(-1)}\right\}$,

$$
D_{\rho}^{m}\left(\theta_{0}(\rho) \mp 1\right)=O\left(e^{-\alpha|\rho|}\right) \quad \text { as } \pm \rho \rightarrow \infty, \quad \forall m \in \mathbb{N} .
$$

Let $\theta_{1} \in C^{1}(\mathbb{R}) \cap L^{\infty}(\mathbb{R})$ be any function satisfying

$$
\int_{\mathbb{R}} \theta_{0}^{\prime 2} f^{\prime \prime}\left(\theta_{0}\right) \theta_{1}=0 .
$$

Let $\Omega^{-} \subset \subset \Omega$ be a subset with $C^{3}$ boundary $\gamma=\partial \Omega^{-}$. Denote by $d(x)$ the signed distance (negative in $\Omega^{-}$) from $x$ to $\gamma$, and by $s(x)$, for $x$ close to $\gamma$, the projection from $x$ on $\gamma$ along the normal to $\gamma$. by

We look for the spectrum of the linearized operator of $-\Delta u-\varepsilon^{-2} f^{\prime}(u)$ around $u=\psi^{\varepsilon}$ given

$$
\psi^{\varepsilon}(x)= \begin{cases}\theta_{0}(d(x) / \varepsilon)+\varepsilon p^{\varepsilon}(s(x)) \theta_{1}(d(x) / \varepsilon)+O(1) \varepsilon^{2} & \text { if }|d(x)| \leqslant \sqrt{\varepsilon} \\ \pm 1+O(1) \varepsilon & \text { if } \pm d(x) \geqslant \sqrt{\varepsilon}\end{cases}
$$

We use the following spectrum estimate.

Proposition 1 Let $\gamma \in C^{3}$, and $p^{\varepsilon}$ and $O(1)$ in $(16)$ be bounded independently of $\varepsilon$. Then there exists a positive constant $C^{*}$ depending on $\|\gamma\|_{C^{3}},\left\|p^{\varepsilon}\right\|_{L^{\infty}}$ and $\|O(1)\|_{L^{\infty}}$ such that for every $\varepsilon \in(0,1]$ and $\phi \in H^{1}(\Omega)$,

$$
\int_{\Omega}\left\{|\nabla \phi|^{2}-\varepsilon^{-2} f^{\prime}\left(\psi^{\varepsilon}\right) \phi^{2}\right\} \geqslant-C^{*} \int_{\Omega} \phi^{2} .
$$

This inequality is established in [7]. Note that such a spectrum estimate was proven by de Mottoni and Schatzman in [10], but around a different approximation $\psi_{\varepsilon}$. A unified treatment of the spectra was later obtained in [7] in a more general situation and has been used in [1, 8]. Let us also mention previous results in this direction obtained in [4] and also in [2] for the 2D Cahn-Hilliard equation.

We define the linearized operator around $\theta_{0}(\rho)$ acting on $v=v(\rho)$ by

$$
\mathcal{L} v:=-v^{\prime \prime}-f^{\prime}\left(\theta_{0}\right) v
$$

In our application, $\theta_{1}$ is the unique solution to

$$
\begin{aligned}
\mathcal{L} \theta_{1} & =1-\sigma \theta_{0}^{\prime} \quad \text { in } \mathbb{R}, \\
\theta_{1}(0) & =0, \quad \sigma:=2 / \int_{\mathbb{R}} \theta_{0}^{\prime 2} .
\end{aligned}
$$

Integrating $\theta_{0}^{\prime \prime} \mathcal{L} \theta_{1}$ by parts over $\mathbb{R}$, one can verify that $(15)$ is satisfied. 
We remark that the distance function $d$ in $(16)$ can be replaced by a "quasi-distance" function $d^{\varepsilon}$ given by

$$
d^{\varepsilon}(x)=d(x)-\varepsilon h_{1}(s(x))-\varepsilon^{2} h_{2}(s(x))+O(1) \varepsilon^{3}
$$

where $h_{1}$ and $h_{2}$ are smooth functions of $s \in \gamma$.

\subsection{Solvability condition}

Lemma 3 Assume that $f$ satisfies (2). Let $\theta_{0}$ be the solution to (14), define $\alpha=$ $\min \left\{\sqrt{-f^{\prime}(1)}, \sqrt{-f^{\prime}(-1)}\right\}$ and let $\mathcal{L}$ be defined in $(17)$. Assume that a function $h(\rho, s, t)$ satisfies, as $\rho \rightarrow \pm \infty$,

$$
D_{\rho}^{m} D_{s}^{n} D_{t}^{l}\left[h(\rho, s, t)-h^{ \pm}(t)\right]=O\left(|\rho|^{i} e^{-\alpha|\rho|}\right)
$$

for some $i \geqslant 0$ and all $(m, n, l) \in \mathbb{N}^{3}$ and $(s, t)$ in $U \times[0, T]$, where $U \subset \mathbb{R}^{n-1}$. Then

$$
\mathcal{L} Q=h(\cdot, s, t) \quad \text { in } \mathbb{R}, \quad Q(0, s, t)=0,
$$

has a unique bounded solution $Q(\rho, s, t)$ if and only if

$$
\forall(s, t) \in U \times[0, T], \quad \int_{\mathbb{R}} h(\rho, s, t) \theta_{0}^{\prime}(\rho) d \rho=0 .
$$

If the solution exists, then it satisfies, for all $(m, n, l) \in \mathbb{N}^{3}$ and $(s, t) \in U \times[0, T]$,

$$
D_{\rho}^{m} D_{s}^{n} D_{t}^{l}\left[Q(\rho, s, t)+\frac{h^{ \pm}(t)}{f^{\prime}( \pm 1)}\right]=O\left(|\rho|^{i} e^{-\alpha|\rho|}\right) \quad \text { as } \rho \rightarrow \pm \infty .
$$

Proof. Note that $\mathcal{L} \theta_{0}^{\prime}=0$ due to translation invariance, and that the null-space of $\mathcal{L}$ is spanned by $\theta_{0}^{\prime}$. Thus the ode $\mathcal{L} Q=h$ can be solved explicitly assuming that $h$ satisfies the condition 19 . We omit the details of the proof; see [10, 7, 1].

\section{Differential geometry: local coordinates}

\subsection{Parametrization around the limit interface}

Let $\Gamma:=\bigcup_{t \in[0, T]}\left(\gamma_{t} \times\{t\}\right) \subset \Omega_{T}$ be the smooth solution to [4] on $[0, T]$ with $\left.\gamma_{t}\right|_{t=0}=\gamma_{0}$ satisfying (5). Let $\Omega_{t} \subset \subset \Omega$ be the domain enclosed by $\gamma_{t}$, with $\gamma_{t}=\partial \Omega_{t}$. For each fixed $t \in[0, T]$, we use $d(x, t)$ to denote the signed distance from $x$ to $\gamma_{t}$ (negative in $\Omega_{t}$ ). Then $d(\cdot, \cdot)$ is smooth in a tubular neighborhood of the interface. We choose a parametrization of $\gamma_{t}$ by $X_{0}(s, t)$ with $s \in U \subset \mathbb{R}^{n-1}$ so that

$$
\left(\frac{\partial X_{0}}{\partial s_{1}}, \ldots, \frac{\partial X_{0}}{\partial s_{n-1}}\right)
$$

is a basis of the tangent space to $\gamma_{t}$ at $X_{0}(s, t)$, for each $s \in U$. We denote by $\mathbf{n}(s, t)$ the unit outer normal vector on $\partial \Omega_{t}=\gamma_{t}$ so that

$$
\mathbf{n}(s, t)=\nabla d\left(X_{0}(s, t), t\right) .
$$

Up to a suitable multiplication factor $s_{1} \rightarrow \lambda s_{1}$, we may assume that

$$
\operatorname{det}\left(\mathbf{n}(s, t), \frac{\partial X_{0}}{\partial s_{1}}, \ldots, \frac{\partial X_{0}}{\partial s_{n-1}}\right)=1
$$


Next for each fixed $t \in[0, T]$, a local parametrization by coordinates $(s, r) \in U \times(-3 \delta, 3 \delta)$ is obtained by

$$
x=X_{0}(s, t)+r \mathbf{n}(s, t)=X(r, s, t),
$$

which defines a local diffeomorphism from $(-3 \delta, 3 \delta) \times U$ onto the tubular neighbourhood of $\gamma_{t}$,

$$
V_{3 \delta}^{t}=\{x \in \Omega:|d(x, t)|<3 \delta\} .
$$

We denote the inverse by

$$
r=d(x, t), \quad s=\mathbf{S}(x, t)=\left(S^{1}(x, t), \ldots, S^{n-1}(x, t)\right) .
$$

In particular, since for all fixed $s \in U, t \in[0, T]$ and for all $r \in(-3 \delta, 3 \delta)$,

$$
d\left(X_{0}(s, t)+r \mathbf{n}(s, t), t\right)=r
$$

it follows by differentiation with respect to $r$ that for all $r \in(-3 \delta, 3 \delta)$,

$$
\nabla d\left(X_{0}(s, t)+r \mathbf{n}(s, t), t\right) \cdot \mathbf{n}(s, t)=1 .
$$

Since

$$
|\nabla d(x, t)|=1 \quad \text { for } x \text { close to } \gamma_{t},
$$

this equality imposes that for all $(r, s) \in(-3 \delta, 3 \delta) \times U$,

$$
\nabla d\left(X_{0}(s, t)+r \mathbf{n}(s, t), t\right)=\mathbf{n}(s, t),
$$

proving that $\nabla d$ is constant along the normal lines to $\gamma_{t}$. Thus the projection $\mathbf{S}(x, t)$ from $x$ on $\gamma_{t}$ is defined by

$$
X_{0}(\mathbf{S}(x, t), t)=x-d(x, t) \nabla d(x, t) .
$$

It also follows from 25 that for all $i=1, \ldots, n$ and for $x \in V_{3 \delta}^{t}$,

$$
\sum_{j=1}^{n} \frac{\partial^{2} d}{\partial x_{i} \partial x_{j}}(x, t) \frac{\partial d}{\partial x_{j}}(x, t)=0 .
$$

Thus the symmetric matrix $D_{x}^{2} d(x, t)$ has eigenvalues $\left\{\kappa_{1}, \ldots, \kappa_{n-1}, 0\right\}$ with unit eigenvectors $\left\{\tau_{1}, \ldots, \tau_{n-1}, \nabla d\right\}$ forming an orthonormal basis of $\mathbb{R}^{n}$ for $x \in V_{3 \delta}^{t}$. In particular, for $x \in \gamma_{t}$, the $\tau_{i}$ are the principal directions and the $\kappa_{i}$ are the principal curvatures of $\gamma_{t}$. Note that $\left\{\tau_{1}, \ldots, \tau_{n-1}\right\}$ form a basis of the tangent hyperplane to $\gamma_{t}$ at $x=X_{0}(s, t)$. By definition, $K$ and $K_{\gamma_{t}}$ are respectively the sum of the principal curvatures and the mean curvature of $\gamma_{t}$, given by

$$
K=(n-1) K_{\gamma_{t}}=\Delta d\left(X_{0}(s, t), t\right)=\sum_{i=1}^{n-1} \kappa_{i}(s, t) .
$$

Note that using (28), for $x \in \gamma_{t}$, we have

$$
\begin{aligned}
\nabla d \cdot \nabla \Delta d & =\sum_{i j} \frac{\partial}{\partial x_{j}}\left(\frac{\partial d}{\partial x_{i}} \frac{\partial^{2} d}{\partial x_{i} \partial x_{j}}\right)-\sum_{i j}\left(\frac{\partial^{2} d}{\partial x_{i} \partial x_{j}}\right)^{2} \\
& =-\sum_{i j}\left(\frac{\partial^{2} d}{\partial x_{i} \partial x_{j}}\right)^{2}=-\operatorname{Trace}\left(\left(D_{x}^{2} d\right)^{2}\right)=-\sum_{i=1}^{n-1} \kappa_{i}^{2}
\end{aligned}
$$


We denote

$$
b(s, t)=-\left.\nabla d \cdot \nabla \Delta d\right|_{X_{0}(s, t), t}=\sum_{i=1}^{n-1} \kappa_{i}^{2}(s, t) .
$$

Let $V(s, t)$ be the normal velocity of the interface at the point $X_{0}(s, t)$ defined by

$$
V(s, t)=\left(X_{0}\right)_{t}(s, t) \cdot \mathbf{n}(s, t) .
$$

Using 26, we have

$$
V(s, t)=\left(X_{0}\right)_{t}(s, t) \cdot \nabla d\left(X_{0}(s, t)+r \mathbf{n}(s, t), t\right)=-d_{t}(X(r, s, t), t),
$$

where the last equality follows from differentiating with respect to $t$ the identity

$$
d\left(X_{0}(s, t)+r \mathbf{n}(s, t), t\right)=r .
$$

It follows that $d_{t}(x, t)$ is independent of $r=d(x, t)$ for $|r|$ small enough. Changing coordinates from $(x, t)$ to $(r, s, t)$, we associate to any function $\phi(x, t)$ the function

$$
\tilde{\phi}(r, s, t)=\phi\left(X_{0}(s, t)+r \mathbf{n}(s, t), t\right)
$$

or equivalently

$$
\phi(x, t)=\tilde{\phi}(d(x, t), \mathbf{S}(x, t), t) .
$$

By differentiation we obtain the formulas

$$
\begin{aligned}
\partial_{t} \phi & =\left(-V \partial_{r}+\partial_{t}^{\Gamma}\right) \tilde{\phi} \\
\nabla \phi & =\left(\mathbf{n} \partial_{r}+\nabla^{\Gamma}\right) \tilde{\phi} \\
\Delta \phi & =\left(\partial_{r r}+\Delta d \partial_{r}+\Delta^{\Gamma}\right) \tilde{\phi}
\end{aligned}
$$

with

$$
\begin{aligned}
\partial_{t}^{\Gamma} \tilde{\phi} & =\left(\partial_{t}+\sum_{i=1}^{n-1} S_{t}^{i} \partial_{s^{i}}\right) \tilde{\phi} \\
\nabla^{\Gamma} \tilde{\phi} & =\left(\sum_{i=1}^{n-1} \nabla S^{i} \partial_{s^{i}}\right) \tilde{\phi}, \\
\Delta^{\Gamma} \tilde{\phi} & =\left(\sum_{i=1}^{n-1} \Delta S^{i} \partial_{s^{i}}+\sum_{i, j=1}^{n-1} \nabla S^{i} \cdot \nabla S^{j} \partial_{s^{i} s^{j}}\right) \tilde{\phi},
\end{aligned}
$$

where $\nabla S^{i}, S_{t}^{i}, \Delta d, d_{t}$ are evaluated at $x=X(r, s, t)$ and are viewed as functions of $(r, s, t)$. Note that the mixed derivatives of the form $\partial_{r s^{j}}^{2} \tilde{\phi}$ do not appear eventually in 33 because for all $j=1, \ldots, n-1$ and $x \in V_{3 \delta}^{t}$,

$$
\nabla S^{j}(x, t) \cdot \nabla d(x, t)=0 .
$$

(This follows from differentiating with respect to $r$ the identity

$$
\forall r \in(-3 \delta, 3 \delta), \quad S^{j}\left(X_{0}(s, t)+r \mathbf{n}(s, t), t\right)=s^{j},
$$

which holds for all fixed $s \in U, t \in[0, T]$ and $j=1, \ldots, n-1$.) 


\subsection{The stretched variable}

Following the method used in [9], we now define the stretched variable $\rho$ by considering a graph over $\gamma_{t}$ of the form

$$
\tilde{\gamma}_{t}^{\varepsilon}=\left\{X(r, s, t): r=\varepsilon h_{\varepsilon}(s, t), s \in U\right\},
$$

which is (formally) expected to be a representation of the 0-level set at time $t$ of the solution $u^{\varepsilon}$ of Problem $\left(P^{\varepsilon}\right)$.

The stretched variable $\rho$ is then defined by

$$
\rho=\rho^{\varepsilon}(x, t)=\frac{d(x, t)-\varepsilon h_{\varepsilon}(\mathbf{S}(x, t), t)}{\varepsilon},
$$

which represents the distance from $x$ to $\tilde{\gamma}_{t}^{\varepsilon}$ in the normal direction divided by $\varepsilon$. From now on, we use $(\rho, s, t)$ as independent variables for the inner expansions. The relation between the old and new variables is

$$
x=\hat{X}(\rho, s, t)=X\left(\varepsilon\left(\rho+h_{\varepsilon}(s, t)\right), s, t\right)=X_{0}(s, t)+\varepsilon\left(\rho+h_{\varepsilon}(s, t)\right) \mathbf{n}(s, t) .
$$

We associate to any function $w(x, t)$ the function

$$
\hat{w}(\rho, s, t)=w\left(X_{0}(s, t)+\varepsilon\left(\rho+h_{\varepsilon}(s, t)\right) \mathbf{n}(s, t), t\right)
$$

or equivalently

Note that

$$
w(x, t)=\hat{w}\left(\frac{d(x, t)-\varepsilon h_{\varepsilon}(\mathbf{S}(x, t), t)}{\varepsilon}, \mathbf{S}(x, t), t\right) .
$$

$$
\tilde{w}(r, s, t)=\hat{w}\left(\frac{r-\varepsilon h_{\varepsilon}(s, t)}{\varepsilon}, s, t\right) .
$$

The relationship between $w, \tilde{w}, \hat{w}$ is summarized as follows:

$$
w(x, t)=\tilde{w}\left(\varepsilon \rho+\varepsilon h_{\varepsilon}, s, t\right)=\hat{w}(\rho, s, t)=\hat{w}\left(\frac{d-\varepsilon h_{\varepsilon}(s, t)}{\varepsilon}, s, t\right) .
$$

In view of (33), we obtain the following formulas for differentiation:

$$
\begin{aligned}
\partial_{t} w & =\left(-V \varepsilon^{-1}-\partial_{t}^{\Gamma} h_{\varepsilon}\right) \hat{w}_{\rho}+\partial_{t}^{\Gamma} \hat{w}, \\
\nabla w & =\left(\mathbf{n} \varepsilon^{-1}-\nabla^{\Gamma} h_{\varepsilon}\right) \hat{w}_{\rho}+\nabla^{\Gamma} \hat{w}, \\
\Delta w & =\left(\varepsilon^{-2}+\left|\nabla^{\Gamma} h_{\varepsilon}\right|^{2}\right) \hat{w}_{\rho \rho}+\left(\Delta d \varepsilon^{-1}-\Delta^{\Gamma} h_{\varepsilon}\right) \hat{w}_{\rho}-2 \nabla^{\Gamma} h_{\varepsilon} \cdot \nabla^{\Gamma} \hat{w}_{\rho}+\Delta^{\Gamma} \hat{w},
\end{aligned}
$$

where in the above formula for $\Delta w$,

$$
\begin{aligned}
\Delta d & =\left.\Delta d\right|_{x=X_{0}(s, t)+\varepsilon\left(\rho+h_{\varepsilon}(s, t)\right) \mathbf{n}(s, t)} \\
& \approx K(s, t)-\varepsilon\left(\rho+h_{\varepsilon}(s, t)\right) b(s, t)+\sum_{i \geqslant 2} \varepsilon^{i} b_{i}(s, t)\left(\rho+h_{\varepsilon}(s, t)\right)^{i},
\end{aligned}
$$

with $b$ defined in (30), $K$ defined in 29 , and for some given functions $\left(b_{i}(s, t)\right)_{i \geqslant 2}$ only depending on $\gamma_{t}$. Therefore

$$
\begin{aligned}
\varepsilon^{2}\left(\partial_{t} w-\Delta w\right)= & -\hat{w}_{\rho \rho}-\varepsilon(V+\Delta d) \hat{w}_{\rho}+\varepsilon^{2}\left[\left(\partial_{t}^{\Gamma} \hat{w}-\Delta^{\Gamma} \hat{w}\right)-\left(\partial_{t}^{\Gamma} h_{\varepsilon}-\Delta^{\Gamma} h_{\varepsilon}\right) \hat{w}_{\rho}\right] \\
& -\varepsilon^{2}\left[\left|\nabla^{\Gamma} h_{\varepsilon}\right|^{2} \hat{w}_{\rho \rho}-2 \nabla^{\Gamma} h_{\varepsilon} \cdot \nabla^{\Gamma} \hat{w}_{\rho}\right] .
\end{aligned}
$$

The Jacobian. For later purposes, we need to compute the Jacobian of the transformation $\hat{X}$. In the $(\rho, s)$ coordinates, $\mathrm{d} x=\varepsilon J^{\varepsilon}(\rho, s, t) \mathrm{d} s \mathrm{~d} \rho$ where $\varepsilon J^{\varepsilon}(\rho, s, t)=\partial \hat{X}(\rho, s, t) / \partial(\rho, s)$ is the Jacobian. We now prove 
LEMMA 4 For all $\rho \in \mathbb{R}, s \in U$ and $t \in[0, T]$,

$$
J^{\varepsilon}(\rho, s, t)=\prod_{i=1}^{n-1}\left[1+\varepsilon\left(\rho+h_{\varepsilon}(s, t)\right) \kappa_{i}(s, t)\right] .
$$

Proof. The equality (42) is obtained in two steps. First, we consider the function $X=X(r, s, t)$ defined in (22), denote its Jacobian by $J=J(r, s, t)$ and prove that for all $\rho \in \mathbb{R}, s \in U$ and $t \in[0, T]$,

$$
J^{\varepsilon}(\rho, s, t)=J\left(\varepsilon\left(\rho+h_{\varepsilon}(s, t)\right), s, t\right) .
$$

Second, we compute $J$ and show that for all $s \in U$ and all $t \in[0, T]$,

$$
J(r, s, t)=\prod_{i=1}^{n-1}\left[1+r \kappa_{i}(s, t)\right]=1+\Delta d\left(X_{0}(s, t), t\right) r+\sum_{i=2}^{n-1} r^{i} j_{i}(s, t),
$$

for some given functions $j_{i}$ depending on $\gamma_{t}$. Consequently, 42 follows directly from (43) and (44).

In order to establish 43, note that by definition 37,

$$
\hat{X}(\rho, s, t)=X\left(\varepsilon\left(\rho+h_{\varepsilon}(s, t)\right), s, t\right),
$$

so that

and for $i=1, \ldots, n-1$,

$$
\frac{\partial \hat{X}}{\partial \rho}=\varepsilon \frac{\partial X}{\partial r}
$$

$$
\frac{\partial \hat{X}}{\partial s_{i}}=\frac{\partial X}{\partial s_{i}}+\varepsilon \frac{\partial h_{\varepsilon}}{\partial s_{i}} \frac{\partial X}{\partial r} .
$$

Thus for all $\rho \in \mathbb{R}, s \in U$ and $t \in[0, T]$,

$$
\begin{aligned}
\varepsilon J^{\varepsilon}(\rho, s, t) & =\varepsilon \operatorname{det}\left[\frac{\partial X}{\partial r}, \frac{\partial X}{\partial s_{1}}+\varepsilon \frac{\partial h_{\varepsilon}}{\partial s_{1}} \frac{\partial X}{\partial r}, \ldots, \frac{\partial X}{\partial s_{n-1}}+\varepsilon \frac{\partial h_{\varepsilon}}{\partial s_{n-1}} \frac{\partial X}{\partial r}\right] \\
& =\varepsilon \operatorname{det}\left[\frac{\partial X}{\partial r}, \frac{\partial X}{\partial s_{1}}, \ldots, \frac{\partial X}{\partial s_{n-1}}\right]\left(\varepsilon\left(\rho+h_{\varepsilon}(s, t)\right), s, t\right)=\varepsilon J\left(\varepsilon\left(\rho+h_{\varepsilon}(s, t)\right), s, t\right),
\end{aligned}
$$

which is (43).

In order to establish (44), we consider the Hessian matrix of $d$ on $\gamma_{t}$ and denote, for $s \in U$ and $t \in[0, T]$,

so that 28 reads

$$
A=A(s, t)=D_{x}^{2} d\left(X_{0}(s, t), t\right)
$$

$$
A \cdot \mathbf{n}(s, t)=0 .
$$

Moreover, differentiating the identity (26) at $r=0$ with respect to $s_{i}$ for $i=1, \ldots, n-1$ yields

$$
A \cdot \frac{\partial X_{0}}{\partial s_{i}}=\frac{\partial \mathbf{n}}{\partial s_{i}} \text {. }
$$

From

$$
X(r, s, t)=X_{0}(s, t)+r \mathbf{n}(s, t),
$$

it follows, by using (45), that

$$
\frac{\partial X}{\partial r}=\mathbf{n}(s, t)=\left(I_{n}+r A(s, t)\right)(\mathbf{n}(s, t)),
$$


and by (46), for $i=1, \ldots, n-1$,

$$
\frac{\partial X}{\partial s_{i}}=\frac{\partial X_{0}}{\partial s_{i}}+r \frac{\partial \mathbf{n}}{\partial s_{i}}=\left(I_{n}+r A(s, t)\right)\left(\frac{\partial X_{0}}{\partial s_{i}}\right) .
$$

Therefore for all $s \in U$ and $t \in[0, T]$,

$$
\begin{aligned}
J(r, s, t) & =\operatorname{det}\left[\frac{\partial X}{\partial r}, \frac{\partial X}{\partial s_{1}}, \ldots, \frac{\partial X}{\partial s_{n-1}}\right] \\
& =\operatorname{det}\left[\left(I_{n}+r A\right)(\mathbf{n}),\left(I_{n}+r A\right)\left(\frac{\partial X_{0}}{\partial s_{1}}\right), \ldots,\left(I_{n}+r A\right)\left(\frac{\partial X_{0}}{\partial s_{n-1}}\right)\right] \\
& =\operatorname{det}\left[I_{n}+r A(s, t)\right] \operatorname{det}\left[\mathbf{n}, \frac{\partial X_{0}}{\partial s_{1}}, \ldots, \frac{\partial X_{0}}{\partial s_{n-1}}\right],
\end{aligned}
$$

which in view of 21 proves that

$$
J(r, s, t)=\operatorname{det}\left[I_{n}+r A(s, t)\right],
$$

which yields (44), since the eigenvalues of $A(s, t)$ are $\kappa_{1}, \ldots, \kappa_{n-1}, 0$.

\section{The approximate solution}

\subsection{Asymptotic expansions}

Let $k>\max \{2, n / 2\}$ be a fixed integer. In what follows, we use $\approx$ to represent asymptotic expansion: $\phi^{\varepsilon} \approx \sum_{i \geqslant 0} \varepsilon^{i} \phi_{i}$ means that for every integer $j \in \mathbb{N}$, we have $\phi^{\varepsilon}=\sum_{i=0}^{j} \varepsilon^{i} \phi_{i}+O(1) \varepsilon^{j+1}$ where $O(1)$ is bounded independently of $\varepsilon \in(0,1)$. For example, since $f$ is smooth, for any bounded sequence $\left\{b, a_{0}, a_{1}, a_{2}, \ldots\right\}$, we have the asymptotic expansion

$$
\begin{aligned}
f\left(b+\varepsilon \sum_{i \geqslant 0} \varepsilon^{i} a_{i}\right) & \approx \sum_{j \geqslant 0} \varepsilon^{j} f^{(j)}(b)\left(\sum_{i \geqslant 0} \varepsilon^{i} a_{i}\right)^{j} / j ! \\
& \approx f(b)+\varepsilon f^{\prime}(b) \sum_{i \geqslant 0} \varepsilon^{i} a_{i}+\varepsilon^{2} \sum_{i \geqslant 0} \varepsilon^{i} f_{i}\left(b, a_{0}, \ldots, a_{i}\right),
\end{aligned}
$$

where for any fixed $b, f_{i}\left(b, a_{0}, \ldots, a_{i}\right)$ is a polynomial in $\left(a_{0}, \ldots, a_{i}\right)$ of degree $\leqslant i+2$.

Outer expansion. We expand $\lambda^{\varepsilon}(t)$ and $u^{\varepsilon}(x, t)$ for $|d(x, t)| \geqslant 3 \delta$ as

$$
\begin{aligned}
\lambda^{\varepsilon}(t) & \approx \lambda_{0}(t)+\varepsilon \lambda_{1}(t)+\varepsilon^{2} \lambda_{2}(t)+\cdots, \\
u^{\varepsilon}(x, t) & \approx u_{\varepsilon}^{ \pm}(t):= \pm 1+\varepsilon\left\{u_{0}^{ \pm}(t)+\varepsilon u_{1}^{ \pm}(t)+\cdots\right\} .
\end{aligned}
$$

Substituting (48) and (49) into (7) gives

$$
f\left(u_{\varepsilon}^{ \pm}(t)\right)=\varepsilon \lambda^{\varepsilon}(t)+\varepsilon^{2}\left(u_{\varepsilon}^{ \pm}\right)^{\prime}(t),
$$

which yields, for all $i \geqslant 0$,

$$
u_{i}^{ \pm}(t)=\left\{\lambda_{i}-f_{i-1}\left( \pm 1, u_{0}^{ \pm}, \ldots, u_{i-1}^{ \pm}\right)+\left(u_{i-2}^{ \pm}\right)^{\prime}(t)\right\} / f^{\prime}( \pm 1),
$$

where $f_{-1}=u_{-2}^{ \pm}=0, u_{-1}^{ \pm}= \pm 1$, and $f_{i}(i \geqslant 0)$ is defined in 47 . Hence, $u_{i}^{ \pm}$are determined by $\left\{\lambda_{0}, \ldots, \lambda_{i}\right\}$. 
Inner expansion. We shall assume that $h_{\varepsilon}$ has the asymptotic expansion

$$
\varepsilon h_{\varepsilon}(s, t) \approx \varepsilon h_{1}(s, t)+\varepsilon^{2} h_{2}(s, t)+\cdots, \quad(s, t) \in U \times[0, T] .
$$

Near the interface, we assume that the function $\hat{u}^{\varepsilon}$ associated to $u^{\varepsilon}$ by 38 has the asymptotic expansion

$$
\hat{u^{\varepsilon}}(\rho, s, t) \approx \theta_{0}(\rho)+\varepsilon\left\{u_{0}(\rho, s, t)+\varepsilon u_{1}(\rho, s, t)+\cdots\right\} .
$$

In the following, the zeroth order expansion refers to

$$
\left\{d(x, t), \lambda_{0}(t), u_{0}(\rho, s, t), u_{0}^{ \pm}(t)\right\}
$$

and the $i$-th order expansion refers to

$$
\left\{h_{i}(s, t), \lambda_{i}(t), u_{i}(\rho, s, t), u_{i}^{ \pm}(t)\right\} .
$$

We shall use $(\ldots)_{i-1}$ to denote a generic function of $(\rho, s, t)$ depending only on expansions of order $\leqslant i-1$.

Matching condition. We suppose that for all $i \in \mathbb{N}$,

$$
\forall(s, t) \in U \times[0, T], \quad \lim _{\rho \rightarrow \pm \infty} u_{i}(\rho, s, t)=u_{i}^{ \pm}(t) .
$$

Translation. We also impose, for all $i \in \mathbb{N}$,

$$
\forall(s, t) \in U \times[0, T], \quad u_{i}(0, s, t)=0,
$$

to be consistent with the assumption that $\rho=0$ is the 0 -level set of $u^{\varepsilon}$.

\subsection{The u-equation in the new variables}

The equation (7) reads

$$
-f(u)=-\varepsilon^{2}\left(u_{t}-\Delta u\right)-\varepsilon \lambda_{\varepsilon}(t) .
$$

In the new variables $(\rho, s, t)$, using 41 , it becomes the following equation for the function $u=\hat{u}^{\varepsilon}$ associated to $u^{\varepsilon}$ by 38 :

$$
\begin{aligned}
-f(u)= & u_{\rho \rho}+\varepsilon\left[(V(s, t)+\Delta d) u_{\rho}-\lambda_{\varepsilon}\right]+\varepsilon^{2}\left[\left(\Delta^{\Gamma} u-\partial_{t}^{\Gamma} u\right)+\left(\partial_{t}^{\Gamma} h_{\varepsilon}-\Delta^{\Gamma} h_{\varepsilon}\right) u_{\rho}\right] \\
& +\varepsilon^{2}\left[\left|\nabla^{\Gamma} h_{\varepsilon}\right|^{2} u_{\rho \rho}-2 \nabla^{\Gamma} h_{\varepsilon} \cdot \nabla^{\Gamma} u_{\rho}\right],
\end{aligned}
$$

where $V(s, t)$ is given by (31) and $\Delta d$ is expanded using (40) and (51) as

$$
\Delta d \approx K(s, t)-\sum_{i \geqslant 1} \varepsilon^{i}\left[b(s, t) h_{i}(s, t)+\delta_{i-1}(\rho, s, t)\right],
$$

with $\delta_{i-1}$ depending only on expansions of order $\leqslant i-1$ (in particular, $\delta_{0}(\rho, s, t)=\rho b(s, t)$ ). Note that $\delta_{i-1}(\rho, s, t)$ is a polynomial in $\rho$ of degree $\leqslant i$, whose coefficients are polynomials in $\left(h_{1}, \ldots, h_{i-1}\right)$ with $(s, t)$-dependent coefficients. 


\subsection{The recursive $i$-th equations}

The zeroth order expansion. Since $\theta_{0}$ defined in (14) satisfies

$$
-f\left(\theta_{0}\right)=\left(\theta_{0}\right)_{\rho \rho}, \quad \theta_{0}( \pm \infty)= \pm 1, \quad \theta_{0}(0)=0,
$$

the equation (55) is satisfied at zeroth order as also are the matching and translation conditions (53)-(54).

The first order expansion. At first order $\left(\varepsilon^{1}\right)$, the equation 55 imposes

$$
\mathcal{L} u_{0}=(K(s, t)+V(s, t)) \theta_{0}^{\prime}(\rho)-\lambda_{0}(t),
$$

with $\mathcal{L}$ defined in $(17)$. The solvability condition stated in Lemma 3 reads

$$
(K(s, t)+V(s, t)) \int_{\mathbb{R}}\left(\theta_{0}^{\prime}\right)^{2}(z) \mathrm{d} z=2 \lambda_{0}(t),
$$

or, by definition of $\sigma$ in $(18)$,

$$
V(s, t)=-K(s, t)+\sigma \lambda_{0}(t) \quad \text { for } s \in U,
$$

also equivalent in view of 31 to

$$
d_{t}=\Delta d-\sigma \lambda_{0}(t) \quad \text { on } \gamma_{t}
$$

Moreover equation (57) has then a unique solution satisfying (53)-54 which is given by

$$
u_{0}(\rho, s, t)=-\lambda_{0}(t) \theta_{1}(\rho)
$$

for all $(s, t) \in U \times[0, T]$. Note that for all non-negative $m, n, l$,

$$
D_{\rho}^{m} D_{s}^{n} D_{t}^{l}\left[u_{0}(\rho, s, t)-u_{0}^{ \pm}(t)\right]=O\left(e^{-\alpha|\rho|}\right) \quad \text { as } \rho \rightarrow \pm \infty .
$$

Higher order expansion. Plugging the expansions (47), (51), 52) into (55) and using (58) and (56) leads to the identity

$$
\begin{aligned}
-f\left(\theta_{0}\right)-\varepsilon f^{\prime}\left(\theta_{0}\right)\left(\sum_{i \geqslant 0} \varepsilon^{i} u_{i}\right)-\varepsilon^{2} \sum_{i \geqslant 0} \varepsilon^{i} f_{i}\left(\theta_{0}, u_{0}, \ldots, u_{i}\right) \\
=\theta_{0}^{\prime \prime}+\varepsilon\left(\sum_{i \geqslant 0} \varepsilon^{i}\left(u_{i}\right)_{\rho \rho}\right)+\varepsilon\left[\left(\sigma \lambda_{0}(t)-\sum_{i \geqslant 1} \varepsilon^{i}\left(b h_{i}+\delta_{i-1}\right)\right) u_{\rho}-\sum_{i \geqslant 0} \varepsilon^{i} \lambda_{i}\right] \\
+\varepsilon^{3} \sum_{i \geqslant 0} \varepsilon^{i}\left(\Delta^{\Gamma}-\partial_{t}^{\Gamma}\right) u_{i}-\varepsilon\left(\sum_{i \geqslant 1} \varepsilon^{i}\left(\Delta^{\Gamma}-\partial_{t}{ }^{\Gamma}\right) h_{i}\right)\left(\theta_{0}^{\prime}+\varepsilon \sum_{i \geqslant 0} \varepsilon^{i}\left(u_{i}\right)_{\rho}\right) \\
+\left[\varepsilon^{2}\left|\nabla^{\Gamma} h_{\varepsilon}\right|^{2} u_{\rho \rho}-2 \varepsilon\left(\sum_{i \geqslant 1} \varepsilon^{i} \nabla^{\Gamma} h_{i}\right) \cdot \nabla^{\Gamma} u_{\rho}\right] .
\end{aligned}
$$

Define the operator $\mathcal{N}^{\Gamma}$ acting on functions $h=h(s, t)$ by

$$
\mathcal{N}^{\Gamma} h:=\partial_{t}^{\Gamma} h-\Delta^{\Gamma} h-b h .
$$

We derive below the $(i+1)$-th order expansion for $i \geqslant 1$ and obtain the following result. 
LEMma 5 The cancellation of the term of order $\varepsilon^{i+1}$, with $i \geqslant 1$, in 55 is equivalent to

$$
\mathcal{L} u_{i}=\mathcal{N}^{\Gamma}\left(h_{i}\right) \theta_{0}^{\prime}-\lambda_{i}(t)+b_{12}\left(\nabla^{\Gamma} h_{1} \cdot \nabla^{\Gamma} h_{i}\right) \theta_{0}^{\prime \prime}+R_{i-1}(\rho, s, t),
$$

with $R_{i-1}$ only depending on expansions of order $\leqslant i-1$. Moreover $R_{i-1}(\rho, s, t)$ is a polynomial in $\rho$ of degree $\leqslant i$ (whose coefficients are polynomials in $\left(h_{1}, \ldots, h_{i-1}, u_{1}, \ldots, u_{i-1}\right)$ and in their derivatives with respect to $(\rho, s, t))$.

Proof. First note that using 58 , the coefficient of order $\varepsilon^{i+1}$ in 61 is

$$
\begin{aligned}
& \left(u_{i}\right)_{\rho \rho}+\sigma \lambda_{0}(t)\left(u_{i-1}\right)_{\rho}-b(s, t) h_{i}(s, t) \theta_{0}^{\prime}-\lambda_{i}(t)-\delta_{i-1}(s, t) \theta_{0}^{\prime} \\
& \quad=\left(u_{i}\right)_{\rho \rho}-b(s, t) h_{i}(s, t) \theta_{0}^{\prime}-\lambda_{i}(t)+(\ldots)_{i-1},
\end{aligned}
$$

with $(\ldots)_{i-1}$ depending only on expansions of order $\leqslant i-1$. Moreover in view of [56], it is a polynomial in $\rho$ of degree $\leqslant i$ (whose coefficients are polynomials in $\left(h_{1}, \ldots, h_{i-1}, u_{1}, \ldots, u_{i-1}\right)$ and in their derivatives with respect to $(\rho, s, t))$.

Next, in view of 51, the coefficient of order $\varepsilon^{i+1}$ in 62 is

$$
\left(\Delta^{\Gamma}-\partial_{t}^{\Gamma}\right) u_{i-2}+\left(\partial_{t}^{\Gamma}-\Delta^{\Gamma}\right) h_{i} \theta_{0}^{\prime}+(\ldots)_{i-2}=\left(\partial_{t}^{\Gamma}-\Delta^{\Gamma}\right) h_{i} \theta_{0}^{\prime}+(\ldots)_{i-2} .
$$

To obtain the term of order $\varepsilon^{i+1}$ in 63 , note that

$$
\begin{aligned}
\varepsilon^{2}\left|\nabla^{\Gamma} h_{\varepsilon}\right|^{2} & \approx\left|\sum_{i \geqslant 1} \varepsilon^{i} \nabla^{\Gamma} h_{i}\right|^{2} \approx \sum_{i \geqslant 2} \varepsilon^{i}\left(\sum_{j=1}^{i-1} \nabla^{\Gamma} h_{j} \cdot \nabla^{\Gamma} h_{i-j}\right) \\
& \approx \varepsilon^{2}\left|\nabla^{\Gamma} h_{1}\right|^{2}+\sum_{i \geqslant 3} \varepsilon^{i}\left(2 \nabla^{\Gamma} h_{1} \cdot \nabla^{\Gamma} h_{i-1}+(\ldots)_{i-2}\right)
\end{aligned}
$$

so that

$$
\varepsilon^{2}\left|\nabla^{\Gamma} h_{\varepsilon}\right|^{2} u_{\rho \rho} \approx\left[\varepsilon^{2}\left|\nabla^{\Gamma} h_{1}\right|^{2}+\sum_{i \geqslant 3} \varepsilon^{i}\left(2 \nabla^{\Gamma} h_{1} \cdot \nabla^{\Gamma} h_{i-1}+(\ldots)_{i-2}\right)\right]\left[\theta_{0}^{\prime \prime}+\varepsilon \sum_{i \geqslant 0} \varepsilon^{i}\left(u_{i}\right)_{\rho \rho}\right] .
$$

Hence the coefficient of order $\varepsilon^{i+1}$ in $\varepsilon^{2}\left|\nabla^{\Gamma} h_{\varepsilon}\right|^{2} u_{\rho \rho}$ is

$$
b_{1,2}\left(\nabla^{\Gamma} h_{1} \cdot \nabla^{\Gamma} h_{i}\right) \theta_{0}^{\prime \prime}+(\ldots)_{i-2}
$$

with $b_{1,2}=1$ or 2 for $i=1$ or $i \geqslant 2$ respectively.

Similarly, the coefficient of order $\varepsilon^{i+1}$ in the term $-2 \varepsilon^{2} \nabla^{\Gamma} h_{\varepsilon} \cdot \nabla^{\Gamma} u_{\rho}$ is

$$
\nabla^{\Gamma} h_{i-1} \cdot \nabla^{\Gamma}\left(u_{0}\right) \rho+\nabla^{\Gamma} h_{i-2} \cdot \nabla^{\Gamma}\left(u_{1}\right) \rho+\cdots+\nabla^{\Gamma} h_{1} \cdot \nabla^{\Gamma}\left(u_{i-2}\right) \rho
$$

where the first term cancels out since $\nabla^{\Gamma}\left(u_{0}\right)^{\prime}=0$ in view of 60; thus it only depends on expansions of order $\leqslant i-2$, so that the term of order $\varepsilon^{i+1}$ in 63 is given by 68 .

Finally at order $\varepsilon^{i+1}$, with $i \geqslant 1$, using 666- 68), the equation 55] reads

$$
\begin{aligned}
& -f^{\prime}\left(\theta_{0}\right) u_{i}-f_{i-1}\left(\theta_{0}, u_{0}, \ldots, u_{i-1}\right)=\left(u_{i}\right)_{\rho \rho}-\lambda_{i}(t) \\
& +\left(\partial_{t}^{\Gamma} h_{i}-\Delta^{\Gamma} h_{i}-b h_{i}\right) \theta_{0}^{\prime}+b_{12}\left(\nabla^{\Gamma} h_{1} \cdot \nabla^{\Gamma} h_{i}\right) \theta_{0}^{\prime \prime}+(\ldots)_{i-1}(\rho, s, t),
\end{aligned}
$$


which is exactly (65), with $R_{i-1}$ only depending on expansions of order $\leqslant i-1$. Moreover $R_{i-1}(\rho, s, t)$ is a polynomial in $\rho$ of degree $\leqslant i$ as described in Lemma 5 .

The solvability condition. According to Lemma 3 , the equation 65 has a solution if and only if the following solvability condition is satisfied:

$$
\forall(s, t) \in U \times[0, T], \quad \int_{\mathbb{R}} \mathcal{L} u_{i}(\rho, s, t) \theta_{0}^{\prime}(\rho) \mathrm{d} \rho=0 .
$$

Note that

$$
\int_{\mathbb{R}} b_{12}\left(\nabla^{\Gamma} h_{1} \cdot \nabla^{\Gamma} h_{i}\right) \theta_{0}^{\prime \prime}(\rho) \theta_{0}^{\prime}(\rho) \mathrm{d} \rho=b_{12}\left(\nabla^{\Gamma} h_{1} \cdot \nabla^{\Gamma} h_{i}\right)(s, t) \int_{\mathbb{R}} \frac{1}{2}\left[\left(\theta_{0}^{\prime}\right)^{2}\right]^{\prime}(\rho) \mathrm{d} \rho=0,
$$

so that (69) reads

$$
\mathcal{N}^{\Gamma}\left(h_{i}\right)=\sigma \lambda_{i}(t)+r_{i-1}(s, t),
$$

with

$$
r_{i-1}(s, t)=-\frac{\sigma}{2} \int_{\mathbb{R}} R_{i-1}(\rho, s, t) \theta_{0}^{\prime}(\rho) \mathrm{d} \rho
$$

only depending on expansions of order $\leqslant i-1$. We summarize the construction by induction in the next lemma.

LEMma 6 Let $k \geqslant 1$ be given. Assume that for all $i \leqslant k-1$, 65 has a solution $u_{i}$ satisfying

$$
D_{\rho}^{m} D_{s}^{n} D_{t}^{l}\left[u_{i}(\rho, s, t)-u_{i}^{ \pm}(t)\right]=O\left(\rho^{i} e^{-\alpha|\rho|}\right) \quad \text { as } \rho \rightarrow \pm \infty .
$$

Also assume that for $i=k,\left\{h_{i}(s, t), \lambda_{i}(t)\right\}$ satisfies $(70)$. Then for $i=k$, 65) admits a unique solution satisfying $u_{i}(0, s, t)=0$ and (71).

The proof follows from Lemma 3 and an induction argument and is omitted. Just note that in the limit $\rho \rightarrow \pm \infty$, the equation $0=\varepsilon^{2}\left(u_{t}^{\varepsilon}-\Delta u^{\varepsilon}\right)+f\left(u^{\varepsilon}\right)-\left.\varepsilon \lambda^{\varepsilon}\right|_{x=\hat{X}(\rho, s, t)}$ becomes the outer expansion equation, so that $u_{i}( \pm \infty, s, t)=u_{i}^{ \pm}(t)$. Furthermore since $R_{i-1}$ is a polynomial in $\rho$ of degree $\leqslant i, 71$ is satisfied for each $i \geqslant 0$ and $(s, t) \in U \times[0, T]$.

\subsection{Equation for $\lambda^{\varepsilon}$}

To find $\lambda^{\varepsilon}(t)$, we use an asymptotic expansion for $0=\int_{\Omega} u_{t}^{\varepsilon}(x, t) \mathrm{d} x$. We denote by $\Omega_{\varepsilon}^{ \pm}(t)$ the two domains separated by $\tilde{\gamma}_{t}^{\varepsilon}$ defined in $\sqrt{35}$, with $\tilde{\gamma}_{t}^{\varepsilon}=\partial \Omega_{\varepsilon}^{-}(t)$. Hence in view of 36 ,

$$
\begin{aligned}
\Omega_{\varepsilon}^{+}(t) & =\{x \in \Omega: d(x, t)>3 \delta\} \cup\left\{x \in V_{3 \delta}^{t}: d(x, t)-\varepsilon h_{\varepsilon}(\mathbf{S}(x, t), t)>0\right\} \\
& =\{x \in \Omega: d(x, t)>3 \delta\} \cup\left\{x \in V_{3 \delta}^{t}: \rho^{\varepsilon}(x, t)>0\right\}
\end{aligned}
$$

and

$$
\Omega_{\varepsilon}^{-}(t)=\Omega \backslash \overline{\Omega_{\varepsilon}^{+}(t)}=\{x \in \Omega: d(x, t)<-3 \delta\} \cup\left\{x \in V_{3 \delta}^{t}: \rho^{\varepsilon}(x, t)<0\right\} .
$$

We write

$$
\int_{\Omega} u_{t}^{\varepsilon}(x, t) \mathrm{d} x=\int_{|d(x, t)| \geqslant 3 \delta} u_{t}^{\varepsilon}(x, t) \mathrm{d} x+\int_{|d(x, t)|<3 \delta} u_{t}^{\varepsilon}(x, t) \mathrm{d} x
$$


where

$$
\begin{aligned}
\int_{|d(x, t)|<3 \delta} u_{t}^{\varepsilon}(x, t) & \mathrm{d} x \\
= & \int_{\left\{x \in V_{3 \delta}^{t}:\left|\rho^{\varepsilon}(x, t)\right| \geqslant \delta / \varepsilon\right\}} u_{t}^{\varepsilon}(x, t) \mathrm{d} x+\int_{\left\{x \in V_{3 \delta}^{t}:\left|\rho^{\varepsilon}(x, t)\right|<\delta / \varepsilon\right\}} u_{t}^{\varepsilon}(x, t) \mathrm{d} x .
\end{aligned}
$$

In the following we choose $0<\varepsilon \leqslant \varepsilon_{0}$ small enough so that for all $\varepsilon \in\left(0, \varepsilon_{0}\right]$,

$$
\max _{s \in U, t \in[0, T)}\left|\varepsilon h_{\varepsilon}(s, t)\right| \leqslant \delta / 2,
$$

and consequently

$$
\left|\rho^{\varepsilon}(x, t)\right| \geqslant \delta / \varepsilon \Rightarrow|d(x, t)| \geqslant \delta / 2 .
$$

Thus at points $(x, t)$ where either $|d(x, t)| \geqslant 3 \delta$ or $\left|\rho^{\varepsilon}(x, t)\right| \geqslant \delta / \varepsilon$, it follows that $|d(x, t)| \geqslant \delta / 2$, so that

$$
u_{t}^{\varepsilon}(x, t) \approx\left(u_{\varepsilon}^{+}\right)^{\prime}(t) \chi_{\{d(x, t)>0\}}+\left(u_{\varepsilon}^{-}\right)^{\prime}(t) \chi_{\{d(x, t)<0\}}
$$

since exponentially small terms of order $O\left(e^{-\alpha \delta /(2 \varepsilon)}\right)$ do not affect the asymptotic expansion in the $\varepsilon$ power series. Note moreover that if $\left|\rho^{\varepsilon}(x, t)\right| \geqslant \delta / \varepsilon$, then $d(x, t)$ and $\rho=\rho^{\varepsilon}(x, t)$ have the same sign. To simplify the notations, we denote

$$
\begin{aligned}
& \{|\rho| \geqslant \delta / \varepsilon\}=\left\{x \in V_{3 \delta}^{t}:\left|\rho^{\varepsilon}(x, t)\right| \geqslant \delta / \varepsilon\right\}, \\
& \{|\rho|<\delta / \varepsilon\}=\left\{x \in V_{3 \delta}^{t}:\left|\rho^{\varepsilon}(x, t)\right|<\delta / \varepsilon\right\} .
\end{aligned}
$$

Therefore in view of (74)-(75),

$$
\begin{aligned}
\int_{\Omega} u_{t}^{\varepsilon}(x, t) \mathrm{d} x \approx & \int_{|d(x, t)| \geqslant 3 \delta}\left[\left(u_{\varepsilon}^{+}\right)^{\prime}(t) \chi_{\{d>0\}}+\left(u_{\varepsilon}^{-}\right)^{\prime}(t) \chi_{\{d<0\}}\right] \mathrm{d} x \\
& +\int_{|\rho| \geqslant \delta / \varepsilon}\left[\left(u_{\varepsilon}^{+}\right)^{\prime}(t) \chi_{\{\rho>0\}}+\left(u_{\varepsilon}^{-}\right)^{\prime}(t) \chi_{\{\rho<0\}}\right] \mathrm{d} x+\int_{|\rho|<\delta / \varepsilon} u_{t}^{\varepsilon}(x, t) \mathrm{d} x \\
\approx & I_{1}+\int_{|\rho|<\delta / \varepsilon}\left[u_{t}^{\varepsilon}-\left(u_{\varepsilon}^{+}\right)^{\prime}(t) \chi_{\{d(x, t)>0\}}-\left(u_{\varepsilon}^{-}\right)^{\prime}(t) \chi_{\{d(x, t)<0\}}\right] \mathrm{d} x,
\end{aligned}
$$

where

$$
I_{1}=\left(u_{\varepsilon}^{+}\right)^{\prime}(t)\left|\Omega_{\varepsilon}^{+}(t)\right|+\left(u_{\varepsilon}^{-}\right)^{\prime}(t)\left|\Omega_{\varepsilon}^{-}(t)\right| .
$$

In the second integral, we make the change of variables given in (37) and substitute the expression for $u_{t}^{\varepsilon}$ in formula (39) to obtain

$$
\begin{aligned}
\int_{|\rho|<\delta / \varepsilon}\left[u_{t}^{\varepsilon}-\left(u_{\varepsilon}^{+}\right)^{\prime}(t) \chi_{\{\rho>0\}}-\right. & \left.\left(u_{\varepsilon}^{-}\right)^{\prime}(t) \chi_{\{\rho<0\}}\right] \mathrm{d} x \\
= & \int_{0<\rho<\delta / \varepsilon} \partial_{t}^{\Gamma}\left[\hat{u^{\varepsilon}}(\rho, s, t)-u_{\varepsilon}^{+}(t)\right] \varepsilon J^{\varepsilon}(\rho, s, t) \mathrm{d} \rho \mathrm{d} s \\
& +\int_{-\delta / \varepsilon<\rho<0} \partial_{t}^{\Gamma}\left[\hat{u^{\varepsilon}}(\rho, s, t)-u_{\varepsilon}^{-}(t)\right] \varepsilon J^{\varepsilon}(\rho, s, t) \mathrm{d} \rho \mathrm{d} s \\
& +\int_{|\rho|<\delta / \varepsilon}\left(-V \varepsilon^{-1}-\partial_{t}^{\Gamma} h_{\varepsilon}\right) \frac{\partial \hat{u}^{\varepsilon}}{\partial \rho} \varepsilon J^{\varepsilon}(\rho, s, t) \mathrm{d} \rho \mathrm{d} s .
\end{aligned}
$$


Finally,

$$
\int_{\Omega} u_{t}^{\varepsilon}(x, t) \mathrm{d} x \approx I_{1}+I_{2}+I_{3},
$$

where

$$
I_{2}=\int_{|\rho|<\delta / \varepsilon} \partial_{t}^{\Gamma}\left[\hat{u}^{\hat{\varepsilon}}(\rho, s, t)-u_{\varepsilon}^{+}(t) \chi_{\{\rho>0\}}-u_{\varepsilon}^{-}(t) \chi_{\{\rho<0\}}\right] \varepsilon J^{\varepsilon}(\rho, s, t) \mathrm{d} \rho \mathrm{d} s
$$

and

$$
I_{3}=\int_{|\rho|<\delta / \varepsilon}\left(-V \varepsilon^{-1}-\partial_{t}^{\Gamma} h_{\varepsilon}\right) \frac{\partial \hat{u}^{\varepsilon}}{\partial \rho} \varepsilon J^{\varepsilon}(\rho, s, t) \mathrm{d} \rho \mathrm{d} s .
$$

The calculation for $I_{1}$. The boundary of $\Omega_{\varepsilon}^{-}(t)$ is $\tilde{\gamma}_{t}^{\varepsilon}$, which according to 35 is given in local coordinates $(r, s)$ by $r=\varepsilon h_{\varepsilon}(s, t)$. Therefore in view of (44),

$$
\begin{aligned}
\left|\Omega_{\varepsilon}^{-}(t)\right| & =\left|\Omega_{t}\right|+\int_{U} \int_{0}^{\varepsilon h_{\varepsilon}(s, t)} J(r, s, t) \mathrm{d} r \mathrm{~d} s \\
& \approx\left|\Omega_{t}\right|+\sum_{i \geqslant 1} \varepsilon^{i}\left\{\int_{U} h_{i}(s, t) \mathrm{d} s+(\ldots)_{i-1}\right\},
\end{aligned}
$$

where $(\ldots)_{i-1}$ only depends on expansions of order $\leqslant i-1$. Hence

$$
\left|\Omega_{\varepsilon}^{+}(t)\right|=|\Omega|-\left|\Omega_{\varepsilon}^{-}(t)\right| \approx|\Omega|-\left|\Omega_{t}\right|-\sum_{i \geqslant 1} \varepsilon^{i}\left\{\int_{U} h_{i}(s, t) \mathrm{d} s+(\ldots)_{i-1}\right\} .
$$

From the outer expansion (49), it follows that

$$
\left(u_{\varepsilon}^{ \pm}\right)^{\prime}(t) \approx \varepsilon \sum_{i \geqslant 0} \varepsilon^{i}\left(u_{i}^{ \pm}\right)^{\prime}(t) \approx \sum_{i \geqslant 1} \varepsilon^{i}\left(u_{i-1}^{ \pm}\right)^{\prime}(t),
$$

with $\left(u_{i-1}^{ \pm}\right)^{\prime}(t)$ given by 50 and depending only on expansions of order $\leqslant i-1$. Therefore

$$
I_{1}=\left(u_{\varepsilon}^{+}\right)^{\prime}(t)\left|\Omega_{\varepsilon}^{+}(t)\right|+\left(u_{\varepsilon}^{-}\right)^{\prime}(t)\left|\Omega_{\varepsilon}^{-}(t)\right| \approx \sum_{i \geqslant 1} \varepsilon^{i}(\ldots)_{i-1}
$$

where $(\ldots)_{i-1}$ depends only on expansions of order $\leqslant i-1$.

The calculation for $I_{2}$. Using the expression for $\partial_{t}^{\Gamma} \hat{u}^{\varepsilon}$ in formula 34 and 71 , we compute

$$
\begin{aligned}
& \partial_{t} \Gamma\left[\hat{u}^{\varepsilon}(\rho, s, t)-u_{\varepsilon}^{+}(t) \chi_{\{\rho>0\}}-u_{\varepsilon}^{-}(t) \chi_{\{\rho<0\}}\right] \\
& \approx \varepsilon \sum_{i \geqslant 1} \varepsilon^{i} \partial_{t}^{\Gamma}\left[u_{i}(\rho, s, t)-u_{i}^{+}(t) \chi_{\{\rho>0\}}-u_{i}^{-}(t) \chi_{\{\rho<0\}}\right] \\
& \approx \varepsilon \sum_{i \geqslant 1} \varepsilon^{i}\left(\partial_{t}+\sum_{j=1}^{n-1} S_{t}^{j} \partial_{s^{j}}\right)\left[u_{i}(\rho, s, t)-u_{i}^{+}(t) \chi_{\{\rho>0\}}-u_{i}^{-}(t) \chi_{\{\rho<0\}}\right] \\
& \approx \sum_{i \geqslant 2} \varepsilon^{i} O\left(\rho^{i-1} e^{-\alpha|\rho|}\right)
\end{aligned}
$$


with $O\left(\rho^{i-1} e^{-\alpha|\rho|}\right)$ depending only on expansions of order $\leqslant i-1$. Therefore by definition of $I_{2}$ in 82 ,

$$
I_{2} \approx \sum_{i \geqslant 3} \varepsilon^{i}(\ldots)_{i-2},
$$

where $(\ldots)_{i-2}$ depends only on expansions of order $\leqslant i-2$.

The calculation for $I_{3}$. Using the expansions

$$
\begin{gathered}
\frac{\partial \hat{u^{\varepsilon}}}{\partial \rho} \approx \theta_{0}^{\prime}+\varepsilon \sum_{i \geqslant 0} \varepsilon^{i} \frac{\partial u_{i}}{\partial \rho}, \\
-V-\varepsilon \partial_{t}^{\Gamma} h_{\varepsilon}=d_{t}\left(X_{0}(s, t), t\right)-\sum_{i \geqslant 1} \varepsilon^{i} \partial_{t}^{\Gamma} h_{i}
\end{gathered}
$$

and rewriting the expression for $J^{\varepsilon}$ in 42 as

$$
\begin{aligned}
J^{\varepsilon}(\rho, s, t) & =\prod_{i=1}^{n-1}\left[1+\varepsilon\left(\rho+h_{\varepsilon}(s, t)\right) \kappa_{i}(s, t)\right] \\
& \approx 1+\Delta d\left(X_{0}(s, t), t\right) \varepsilon\left(\rho+h_{\varepsilon}(s, t)\right)+\sum_{i \geqslant 2} \varepsilon^{i}(\ldots)_{i-1},
\end{aligned}
$$

with $(\ldots)_{i-1}$ depending only on expansions of order $\leqslant i-1$, we obtain

$$
\begin{aligned}
(-V & \left.-\varepsilon \partial_{t}^{\Gamma} h_{\varepsilon}\right) \frac{\partial \hat{u}^{\varepsilon}}{\partial \rho} J^{\varepsilon}(\rho, s, t) \\
& \approx d_{t}\left(X_{0}(s, t), t\right) \theta_{0}^{\prime}(\rho)+\sum_{i \geqslant 1} \varepsilon^{i} \theta_{0}^{\prime}(\rho)\left(-\partial_{t}^{\Gamma} h_{i}+d_{t}\left(X_{0}(s, t), t\right) h_{i} \Delta d\right)+\sum_{i \geqslant 1} \varepsilon^{i}(\ldots)_{i-1}
\end{aligned}
$$

so that

$$
\begin{aligned}
I_{3} & \approx \int_{U} \int_{\mathbb{R}}\left\{\theta_{0}^{\prime} d_{t}(s, t)+\sum_{i \geqslant 1} \varepsilon^{i}\left[\theta_{0}^{\prime}\left(-\partial_{t}^{\Gamma} h_{i}+d_{t}(s, t) \Delta d(s, t) h_{i}\right)+(\ldots)_{i-1}\right]\right\} \mathrm{d} \rho \mathrm{d} s \\
& \approx 2 \int_{U} d_{t}(s, t) \mathrm{d} s+\sum_{i \geqslant 1} \varepsilon^{i}\left\{2 \int_{U}\left\{-\partial_{t}^{\Gamma} h_{i}+\left(d_{t} \Delta d\right) h_{i}\right\} \mathrm{d} s+(\ldots)_{i-1}\right\} .
\end{aligned}
$$

Finally, substituting $d_{t}$ and $\partial_{t}^{\Gamma} h_{i}$ by 599 and $(70)$, and using $\int_{U} \Delta^{\Gamma} h_{i} \mathrm{~d} s=0$, we obtain

$$
\frac{1}{2} \int_{\Omega} u_{t}^{\varepsilon} \approx \int_{U}\left(\Delta d-\sigma \lambda_{0}\right) \mathrm{d} s+\sum_{i \geqslant 1} \varepsilon^{i}\left\{\int_{U}\left[\left(-b+d_{t} \Delta d\right) h_{i}-\sigma \lambda_{i}\right] \mathrm{d} s+(\ldots)_{i-1}\right\} .
$$

Thus the condition $\int_{\Omega} u_{t}^{\varepsilon} \mathrm{d} x \approx 0$ is equivalent to

$$
\begin{aligned}
\sigma \lambda_{0}(t) & =\overline{\Delta d(\cdot, t)}, \\
\sigma \lambda_{i}(t) & =-\overline{\left[b(\cdot, t)-d_{t}(\cdot, t) \Delta d(\cdot, t)\right] h_{i}(\cdot, t)}+\Lambda_{i-1}(t), \quad i \geqslant 1,
\end{aligned}
$$


where $\Lambda_{i-1}(t)$ depends only on expansions of order $\leqslant i-1$, and

$$
\overline{\phi(\cdot)}:=\frac{1}{|U|} \int_{U} \phi=\frac{1}{\left|\gamma_{t}\right|} \int_{\gamma_{t}} \phi,
$$

using that $J(0, s, t)=1$. Hence we obtain closed systems for $d, h_{1}, \ldots, h_{i}$, namely

$$
\begin{aligned}
d_{t}(s, t) & =\Delta d(s, t)-\overline{\Delta d(s, t)}, \\
\partial_{t}^{\Gamma} h_{i} & =\Delta^{\Gamma} h_{i}+b h_{i}-\overline{\left[b(\cdot, t)-d_{t}(\cdot, t) \Delta(\cdot, t)\right] h_{i}(\cdot, t)}+\Lambda_{i-1}(t)
\end{aligned}
$$

on $U \times[0, T]$.

\subsection{Construction of expansions of arbitrary order}

We can now use induction to construct each order of expansion as follows:

1) Zeroth order. Given a smooth initial interface $\gamma_{0}, 86$ is equivalent to the volume preserving mean curvature flow (4). By the result established in [14], there is a time $T>0$ such that there is a unique smooth solution on a time interval $[0, T]$. Consequently, $\Gamma=\bigcup_{0 \leqslant t \leqslant T}\left(\gamma_{t} \times\{t\}\right)$ and the modified distance function $d$ are well defined. Set $\lambda_{0}(t)$ as in $84, u_{0}(\rho, s, t)$ as in 60, and $u_{0}^{ \pm}(t)=$ $\lambda_{0}(t) / f^{\prime}( \pm 1)$ as in 50,. We obtain the zeroth order expansion $\left\{d(x, t), \lambda_{0}(t), u_{0}(\rho, s, t), u_{0}^{ \pm}(t)\right\}$.

2) Higher order expansion. Fix $i \geqslant 1$. Assume that all expansions of order $\leqslant i-1$ are constructed. Then $\Lambda_{i-1}(t)$ in (87) is known. Since $\gamma_{t}$ is a smooth hypersurface without boundary, it follows from standard parabolic PDE theory [21] that (87) admits a unique smooth solution (assuming an initial condition such as $h_{i}(\cdot, 0)=0$ on $U$ is given). Consequently, we can define $\lambda_{i}(t)$ as in $(85), u_{i}^{ \pm}(t)$ as in 50 and $u_{i}$ as the solution of 65 given by Lemma 6 This gives the $i$-th order expansion $\left\{h_{i}(s, t), \lambda_{i}(t), u_{i}(\rho, s, t), u_{i}^{ \pm}(t)\right\}$ and completes the induction.

\subsection{Construction of the approximate solution}

We now fix an arbitrary positive integer $k>\max (n, 4)$. We construct an approximate solution $u_{k}^{\varepsilon}$ such that Lemma 2 can be applied.

Let $\delta>0$ be a small fixed constant such that $d(x, t)$ is smooth in the $3 \delta$-neighborhood of $\Gamma$, and for each $t \in[0, T], \gamma_{t}$ is a distance at least $3 \delta$ away from $\partial \Omega$. We define

$$
\begin{aligned}
\rho_{k}^{\varepsilon}(x, t) & =\varepsilon^{-1}\left\{d(x, t)-\sum_{i=1}^{k+1} \varepsilon^{i} h_{i}(\mathbf{S}(x, t), t)\right\}, \\
u_{\varepsilon, k}^{\text {in }}(x, t) & =\theta_{0}\left(\rho_{k}^{\varepsilon}\right)+\varepsilon \sum_{i=0}^{k+1} \varepsilon^{i} u_{i}\left(\rho_{k}^{\varepsilon}(x, t), \mathbf{S}(x, t), t\right), \\
u_{\varepsilon, k, \pm}^{\text {out }}(t) & = \pm 1+\varepsilon \sum_{i=0}^{k+1} \varepsilon^{i} u_{i}^{ \pm}(t) \\
\lambda_{k}^{\varepsilon}(t) & =\sum_{i=0}^{k+1} \varepsilon^{i} \lambda_{i}(t)
\end{aligned}
$$

We note that $\rho_{k}^{\varepsilon}$ and $u_{\varepsilon, k}^{\text {in }}$ are smooth in the $3 \delta$-neighborhood of $\Gamma$. 
Now let $\zeta \in C^{\infty}(\mathbb{R})$ be a cut-off function (depending only on $\delta$ ) satisfying

$$
\begin{array}{cl}
\zeta(s)=1 \quad \text { if }|s| \leqslant \delta, & \zeta(s)=0 \quad \text { if }|s|>2 \delta, \\
0 \leqslant s \zeta^{\prime}(s) \leqslant 4 & \text { if } \delta \leqslant|s| \leqslant 2 \delta .
\end{array}
$$

We define the needed approximate solution $u_{k}^{\varepsilon}$ by

$$
\begin{aligned}
& \tilde{u}_{k}^{\varepsilon}(x, t):=\zeta(d(x, t)) u_{k}^{\text {in }}+[1-\zeta(d(x, t))]\left\{u_{\varepsilon, k,+}^{\text {out }} \chi\{d(x, t)>0\}+u_{\varepsilon, k,-}^{\text {out }} \chi\{d(x, t)<0\}\right\}, \\
& u_{k}^{\varepsilon}(x, t):=\tilde{u}_{k}^{\varepsilon}(x, t)+f_{\Omega}\left\{\tilde{u}_{k}^{\varepsilon}(\cdot, 0)-\tilde{u}_{k}^{\varepsilon}(\cdot, t)\right\}
\end{aligned}
$$

for all $(x, t) \in \bar{\Omega} \times[0, T]$.

The admissible initial data $g^{\varepsilon}$ are then defined as in $(12)$. Then by construction $\left(u_{k}^{\varepsilon}, \lambda_{k}^{\varepsilon}\right)$ is an approximation of order $k$ satisfying the assumptions in Lemma 2. Here we just remark that (i) in the set $\{(x, t): \delta \leqslant \pm d(x, t) \leqslant 2 \delta\}$, the limiting behavior 771) guarantees that $u_{k}^{\varepsilon}(x, t)=$ $u_{\varepsilon, k, \pm}^{\text {out }}(t)+O\left(e^{-\alpha \delta /(4 \varepsilon)}\right)$, valid also after differentiation, (ii) $\partial_{n} u_{k}^{\varepsilon}=0$ on $\partial \Omega_{T}$ since $u_{k}^{\varepsilon}$ is a function of $t$ near $\partial \Omega_{T}$, and (iii) the correction

$$
\int_{\Omega}\left\{\tilde{u}_{k}^{\varepsilon}(\cdot, 0)-\tilde{u}_{k}^{\varepsilon}(\cdot, t)\right\}=-\int_{\Omega} \int_{[0, t]}\left(\tilde{u}_{k}^{\varepsilon}\right)_{t}(y, \tau) \mathrm{d} \tau \mathrm{d} y
$$

is of order $O\left(\varepsilon^{k+1}\right)$, valid also after differentiation. The remaining part of the proof follows the same lines as in [1].

This completes the construction of the approximate solution. The proof of Theorem 1 then follows from the conclusion of Lemma 2 by letting $\varepsilon \rightarrow 0$.

\section{Appendix}

Proof of Lemma 1] We first consider the case $n \geqslant 4$ so that $p=4 / n$. The Gagliardo-NirenbergSobolev inequality (see [15, Theorem 2, p. 265]) states that there exists $C>0$ such that for every $R \in H^{1}(\Omega)$,

$$
\|R\|_{L^{2^{*}}} \leqslant C\|R\|_{H^{1}},
$$

with $2^{*}=2 n /(n-2)$. Using the Poincaré-Wirtinger inequality (see [15, Theorem 1, p. 275]), it follows that there exists $C>0$ such that for every $R \in H^{1}(\Omega)$ with $\int_{\Omega} R \mathrm{~d} x=0$,

$$
\|R\|_{L^{2^{*}}} \leqslant C\|\nabla R\|_{L^{2}} .
$$

Using Hölder's inequality, we have

$$
\|R\|_{L^{2+p}}^{2+p}=\int_{\Omega}|R|^{2}|R|^{p} \leqslant\left(\int_{\Omega}|R|^{2 \beta}\right)^{1 / \beta}\left(\int_{\Omega}|R|^{p \beta^{\prime}}\right)^{1 / \beta^{\prime}}
$$

and we choose

$$
\beta=\frac{n}{n-2}=\frac{2^{*}}{2}, \quad \beta^{\prime}=\frac{n}{2}
$$

to obtain

$$
\|R\|_{L^{2+p}}^{2+p} \leqslant\|R\|_{L^{2^{*}}}^{2}\|R\|_{L^{2}}^{p} .
$$


Combined with (88), this yields the inequality

$$
\|R\|_{L^{2+p}}^{2+p} \leqslant C\|R\|_{L^{2}}^{p}\|\nabla R\|_{L^{2}}^{2}
$$

which is the conclusion of Lemma 1

Next we consider the case that $1 \leqslant n \leqslant 3$ so that $p=1$. Schwarz's inequality then gives

$$
\|R\|_{L^{3}}^{3}=\int_{\Omega}|R|^{2}|R| \leqslant\|R\|_{L^{4}}^{2}\|R\|_{L^{2}}
$$

For $n=1,2,3$, by the Sobolev imbedding, $H^{1} \subset L^{4}$, so that there exists $C>0$ such that for every $R \in H^{1}(\Omega)$,

$$
\|R\|_{L^{4}} \leqslant C\|R\|_{H^{1}}
$$

Using again the Poincaré-Wirtinger inequality, we finally deduce that there exists $C>0$ such that for every $R \in H^{1}(\Omega)$ with $\int_{\Omega} R \mathrm{~d} x=0$,

$$
\|R\|_{L^{3}}^{3} \leqslant C\|\nabla R\|_{L^{2}}^{2}\|R\|_{L^{2}}
$$

which concludes the proof of Lemma 1 .

\section{Acknowledgments}

We would like to thank the referees for their careful reading of the manuscript and their constructive remarks.

Part of this work was done during a visit of the first author to the University of Paris-Sud. The author thanks for the support of the National Science Foundation Grant DMS-9971043.

\section{REFERENCES}

1. Alikakos, N. D., Bates, P. W., \& Chen, X. F. Convergence of the Cahn-Hilliard equation to the Hele-Shaw model. Arch. Ration. Mech. Anal. 128 (1994), 165-205. Zbl 0828.35105 MR 1308851

2. Alikakos, N. D., \& Fusco, G. The spectrum of the Cahn-Hilliard operator for generic interface in higher space dimensions. Indiana Univ. Math. J. 42 (1993), 637-674. Zbl 0798.35123 MR 1237062

3. Barles, G., Soner, H. M., \& Souganidis, P. E. Front propagation and phase field theory. SIAM J. Control Optim. 31 (1993), 439-469. Zbl 0785.35049 MR 1205984

4. Bates, P. W., \& Fife, P. C. Spectral comparison principles for the Cahn-Hilliard and phase-field equations and time scales for coarsening. Phys. D 43 (1990), 335-348. Zbl 0706.58074 MR 1067916

5. BRONSARD, L., \& STOTH, B. Volume preserving mean curvature flow as a limit of nonlocal GinzburgLandau equation. SIAM J. Math. Anal. 28 (1997), 769-807. Zbl 0874.35009 MR 1453306

6. CHEN, X. F. Generation and propagation of interfaces for reaction-diffusion equations. J. Differential Equations 96 (1992), 116-141. Zbl 0765.35024 MR 1153311

7. CHEn, X. F. Spectrums for the Allen-Cahn, Cahn-Hilliard, and phase field equations for generic interface. Comm. Partial Differential Equations 19 (1994), 1371-1395. Zbl 0811.35098 MR 1284813

8. Chen, X. F., \& Caginalp, G. Convergence of the phase field model to its sharp interface limits. Eur. J. Appl. Math. 9 (1998), 417-445. Zbl 0930.35024 MR 1643668

9. Chen, X. F., Caginalp, G., \& ECK, C. A rapidly converging phase field model. Discrete Contin. Dynam. Systems 15 (2006), 1017-1034. Zbl 1119.80009 
10. De Mottoni, P., \& Schatzman, M. Development of interfaces in $\mathbb{R}^{n}$. Proc. Roy. Soc. Edinburgh Sect. A 116 (1990), 207-220. Zbl 0725.35009 MR 1084732

11. De Mottoni, P., \& Schatzman, M. Geometrical evolution of developed interfaces. Trans. Amer. Math. Soc. 347 (1995), 1533-1589. Zbl 0840.35010 MR 1672406

12. Elliott, C. M., \& Garcke, H. Existence results for diffusive surface motion laws. Adv. Math. Sci. Appl. 7 (1997), 467-490. Zbl 0876.35050 MR 1454678

13. Escher, J., \& Ito, K. Some dynamic properties of volume preserving curvature driven flows. Math. Ann. 333 (2005), 213-230. Zbl 1083.53059 MR 2169834

14. Escher, J., \& Simonett, G. The volume preserving mean curvature flow near spheres. Proc. Amer. Math. Soc. 126 (1998), 2789-2796. Zbl 0909.53043 MR 1485470

15. Evans, L. C. Partial Differential Equations. Grad. Stud. in Math. 19, Amer. Math. Soc. (2002).

16. Evans, L. C., Soner, H. M., \& Souganidis, P. E. Phase transitions and generalized motion by mean curvature. Comm. Pure Appl. Math. 45 (1992), 1097-1123. Zbl 0801.35045 MR 1177477]

17. Golovaty, D. The volume preserving motion by mean curvature as an asymptotic limit of reactiondiffusion equations. Quart. Appl. Math. 55 (1997), 243-298. Zbl 0878.35059 MR 1447577

18. Henry, D. Geometric Theory of Semilinear Parabolic Equations. Lecture Notes in Math. 840, Springer (1981). Zbl 0456.35001 MR 0610244

19. Huisken, G. The volume preserving mean curvature flow. J. Reine Angew. Math. 382 (1987), 35-48. Zbl 0621.53007 MR 0921165

20. Ilmanen, T. Convergence of the Allen-Cahn equation to Brakke's motion by mean curvature. J. Differential Geom. 38 (1993), 417-461. Zbl 0784.53035 MR 1237490

21. Ladyzhenskaya, O. A., Solonnikov, V. A., \& Uraltseva, N. N. Linear and Quasilinear Equations of Parabolic Type. Transl. Math. Monogr. 23, Amer. Math. Soc. (1967). Zbl 0164.12302 MR 0241822

22. Rubinstein, J., \& STERnberg, P. Nonlocal reaction-diffusion equations and nucleation. IMA J. Appl. Math. 48 (1992), 249-264. Zbl 0763.35051 MR 1167735 